\title{
A DECOMPOSITION APPROACH FOR THE TWO-STAGE STOCHASTIC SUPPLY NETWORK PLANNING IN LIGHT OF THE ROLLING HORIZON PRACTICE
}

\author{
João Flávio de Freitas Almeida ${ }^{1 *}$ and Samuel Vieira Conceição ${ }^{2}$
}

Received February 26, 2020 / Accepted December 13, 2020

\begin{abstract}
Industries conduct the Sales and Operations Planning (S\&OP) to balance demand and supply aligned to business targets. This study aims at proposing a model and an algorithm for the tactical supply chain planning admitting uncertainty and reflecting the peculiar S\&OP aspect of rolling horizon planning. Therefore, a two-stage stochastic programming model is developed and solved via a multi-cut Benders decomposition algorithm. The model and the solution method are evaluated by numerical experiments and a case study. Results show that the optimal supply chain profit is not proportional to demand, in fact, an increase in demand can even decrease the optimal profit due to capacity constraints along the supply chain. Such findings reinforce that profitability and service level are increased with the synergy of the sales team with production, distribution and procurement team on establishing which demand should be satisfied - or not - in each period. The stochastic solution is compared to deterministic approaches.
\end{abstract}

Keywords: sales and operations planning, supply chain planning, stochastic programming, Benders decomposition.

\section{INTRODUCTION}

Supply chains (SC) are dynamic systems that operate in uncertain environments to meet customers' requirements. Marketing uncertainties and the increasing complexity of operations raise further challenges for SC coordination. To cope with these challenges, companies adopt the Sales and Operations Planning (S\&OP), a centralized planning process that improves vertical integration and inter-functional coordination. S\&OP is a business planning process that comprises the coordination of material, financial, and information flows to balance customer demand with supply capabilities by establishing production mix and volume at the tactical level (Tuomikangas \& Kaipia, 2014).

\footnotetext{
*Corresponding author

${ }^{1}$ Departamento de Engenharia Industrial, Universidade Federal de Minas Gerais. Av. Antônio Carlos, 6627, Belo Horizonte, MG, Brazil. E-mail: joao.flavio@dep.ufmg.br - http://orcid.org/0000-0002-3884-217X

2 Departamento de Engenharia Industrial, Universidade Federal de Minas Gerais. Av. Antônio Carlos, 6627, Belo Horizonte, MG, Brazil. E-mail: svieira@dep.ufmg.br - http://orcid.org/0000-0002-8090-264X
} 
S\&OP has its origins in aggregate production planning, introduced in the 1950s, and settled into use in business and academia only by the early 2000s. IT tools and models support the communication and decision-making process supporting tactics and the strategy. Strategy defines the level of data aggregation. The models use aggregate data to set the medium-term tactical SC plan (Buxey, 2003; Thomé et al., 2012; Ba et al., 2018). Every period, decision-makers share activities of procurement, production, distribution, and sales to produce a consensus forecast and to validate the company tactical plan. The process follows a predefined schedule to review customer demand and supply resources creating a revised plan across an agreed rolling horizon. This framework increases the quality of the first-period plan data, which becomes demand requirements included in a fixed-horizon for short-term programs. Although pilot projects can adopt spreadsheets, when the process evolves to a maturity model, optimization tools with sophisticated models are recommended. However, the development of powerful S\&OP tools integrated into financial parameters requires further research (Thomé et al., 2012; Tuomikangas \& Kaipia, 2014).

Empirical studies show that the S\&OP practice impacts positively on operational performance, particularly on plants with complex manufacturing processes (MT Thomé et al., 2014). Case studies in the electronics, oil, and food sector show companies that successfully adopted the S\&OP by a mathematical modeling approach (Wang et al., 2012; Zhulanova \& Zhulanov, 2014; Taşkın et al., 2015; Nemati et al., 2017). The results include the integration of top-managers on the development of a consensus plan, and the enhancement of coordination between financial and activities of procurement, production, and distribution. The models adopt an aggregate demand forecast and encompass all planning periods. The advantage of adopting the aggregate demand forecast is that it will have reduced variance unless all items are perfectly correlated (Hax \& Meal, 1973). However, S\&OP implementation remains difficult and challenging (Pedroso et al., 2016). Companies lack the right managerial tools to achieve the desired outcomes. Besides, the planning problems remain deterministic on the analysis of a single-stage demand scenario, so uncertainty is not properly evaluated.

The idea of incorporating uncertainty in mathematical programming was pioneered by Dantzig (Dantzig, 1955), and the concept of integrating decentralized SC by stage is introduced by Clark (Clark \& Scarf, 1960). Since then, the understanding of uncertainty via stochastic programming for production and inventory planning has progressed (Birge \& Louveaux, 2011; King \& Wallace, 2012; Alem \& Morabito, 2013; Cunha et al., 2017).

Researches have addressed SC planning on a tactical level admitting uncertainty by a two-stage stochastic programming (2SSP) approach (Moraes \& Faria, 2016). In the S\&OP context, applications approach the configure-to-order system (Chen-Ritzo et al., 2010), the chemical industry (Calfa et al., 2015), the forest-based biomass power plant (Shabani \& Sowlati, 2016), and the blood SC (Dillon et al., 2017). Nevertheless, few works had developed models to evaluate a rolling horizon framework, as discussed in mining operations (Carniato \& Camponogara, 2011) and renewable energy power systems (Wang et al., 2020). The inclusion of uncertainty into SC models lead to large-scale problems due to numerous elements presented on each echelon. There- 
fore, decomposition approaches, as Benders' decomposition (BD) (Benders, 1962), can be used. However, such applications have been employed to the capacity expansion problem, on a strategic level, via the stochastic dual dynamic programming algorithm (Thomé et al., 2013).

To date, the proposed models for S\&OP assume that the first planning period has the same level of importance in comparison to complementary planning periods. The first planning period has more information, instead of pure demand forecast. Moreover, considering its importance to the practice, the analysis of the different scenarios simultaneously should hedge the first-period against the uncertainty of the following periods. The current S\&OP planning practice advocates better planning and fixing the first-period plan. However, there is a lack of practical and academic studies that proposes SC planning models approaching the S\&OP rolling horizon framework that sets the best first-period plan based on uncertain scenarios of complementary periods. Besides, few models address uncertainty on tactical SC planning by a 2SSP approach (Shabani \& Sowlati, 2016; Dillon et al., 2017), and BD (You \& Grossmann, 2013; Oliveira et al., 2014; Kayvanfar et al., 2018), and, to the best of our knowledge, no study has proposed a BD algorithm to solve a 2SSP model based on the broader scope of the S\&OP method.

This manuscript aims at evaluating a tactical SC model aligned to S\&OP rolling horizon planning strategy approaching uncertainty by a 2SSP formulation, which leads to a complex problem; and proposing a multi-cut $\mathrm{BD}$ algorithm to reduce the computational solving time of the large-scale problem. The work evaluates the decomposition approach by a numerical experiment and a case study in a flat steel chain. For modeling the steel production technology, the reader is referred to a seminal paper (Fabian, 1958), a survey (Dutta \& Fourer, 2001), and applications (Seong \& Suh, 2012).

The rest of the paper is organized as follows: Section 2 introduces the 2SSP formulation. Section 3 presents a multi-cut BD algorithm developed for solving large-scale problems. In Sections 4 and 5, the model and algorithm are evaluated by a numerical experiment and a case study, respectively. Finally, Section 6 draws conclusions and suggestions for future research.

\section{MATHEMATICAL FORMULATION}

This section proposes a $2 \mathrm{SSP}$ formulation for S\&OP adopting technology constraints to industries that faces uncertainty on product price and demand. The approach enhances the SC model to evaluate flexibility on tactical SC planning (Almeida et al., 2018) and model the planning process uncertainties in second-stage scenarios, such that the practice is responsible for implementing only the first-period results. At the end of every period, the newly available information updates the second-stage scenarios of the following periods on a rolling planning horizon basis.

The 2SSP formulation adopts a classic notation (Birge \& Louveaux, 2011) and consists in maximizing $c^{\top} x+E_{\xi} Q(x, \xi) \mid A x=b, x \geq 0$, where $E_{\xi} Q(x, \xi)=\max q(\xi)^{\top} y(\xi) \mid T(\xi) x+W y(\xi)=$ $h(\xi), y(\xi) \geq 0$. $E_{\xi}$ is the mathematical expectation with respect to $\xi$, and let $\Xi \subseteq \mathfrak{R}^{N}$ be the support of $\xi$, that is, the smallest closed subset in $\mathfrak{R}^{N}$ such that $P\{\xi \in \Xi\}=1$. 
The model represents a four-echelon SC with $\mathscr{P}$ as the set of products consisting of $\mathscr{X}$ raw materials and $\mathscr{Y}$ finished products (i.e. $\mathscr{P}=\mathscr{X} \cup \mathscr{Y}$ ). Let $\mathscr{L}$ be a location in an SC consisting of $\mathscr{F}$ suppliers, $\mathscr{I}$ industrial plants, $\mathscr{H}$ distribution hubs, and $\mathscr{C}$ customers; thus, $\mathscr{L}=\mathscr{F} \cup$ $\mathscr{I} \cup \mathscr{H} \cup \mathscr{C}$. In this four-echelon SC, $\mathscr{F}$ suppliers provide $\mathscr{X}$ raw materials for $\mathscr{I}$ industrial plants. These plants process raw materials on $\mathscr{R}$ resources and make $\mathscr{Y}$ finished products over $\mathscr{T}$ periods to meet the demands of $\mathscr{C}$ customers. The set of products, locations, resources, and time periods are indexed by $p, l, r$ and $t$, respectively. The notation used to formulate: (i) the deterministic and stochastic parameters; (ii) the first and second-stage variables; and (iii) the elements of the objective function (1) and constraints (2)-(48) are described in Tables 1, 2 and 3 respectively.

The 2SSP objective function model for the optimization problem can be stated as follows:

$$
\begin{aligned}
& \max \Psi=R_{1}^{P}-C_{1}^{L}-C_{1}^{F}-C_{1}^{V}-C_{1}^{P}-C_{1}^{S}-C_{1}^{X}+\mathscr{Q}(\alpha, y, r) \\
& \mathscr{Q}(\alpha, y, r)=\sum_{s \in \mathscr{S}} \rho_{s}\left(R_{2}^{P}-C_{2}^{L}-C_{2}^{F}-C_{2}^{V}-C_{2}^{P}-C_{2}^{S}-C_{2}^{X}\right)
\end{aligned}
$$

The objective function to be maximized in Eq. (1) represents the expected profit resulting from the after-tax revenue and the operational costs. $\mathscr{Q}(\alpha, y, r)=\mathbb{E}_{\omega}[\alpha, y, r, \xi]$ represents the expectation of the second-stage scenarios evaluated over all possible realization of uncertain parameters given the decision of $(\alpha, y, r)$, and $\rho_{s}$ is the occurrence probability of each scenario $s$ $\left(\sum_{s \in \mathscr{S}} \rho_{s}=1\right)$. The objective function is subjected to the following constraints:

$$
\begin{aligned}
& s_{l p t}=S_{l p}^{0} \\
& S_{l p t}^{S} \leq s_{l p t} \leq S_{l p t}^{X} \\
& S_{l p t}^{S} \leq s_{l p t s} \leq S_{l p t}^{X} \\
& L_{l p}^{M} r_{l p} \leq A_{l p t}^{R} \\
& L_{l p}^{M} r_{l p t s} \leq A_{l p t}^{R}
\end{aligned}
$$

$$
\begin{aligned}
& \forall l \in(\mathscr{I} \cup \mathscr{H}), p \in \mathscr{P}, t=0 \\
& \forall l \in(\mathscr{I} \cup \mathscr{H}), p \in \mathscr{P}, t=1 \\
& \forall l \in(\mathscr{I} \cup \mathscr{H}), p \in \mathscr{P}, t \in 2 . .|\mathscr{T}|, s \in \mathscr{S} \\
& \forall l \in \mathscr{F}, p \in \mathscr{X}, t=1 \\
& \forall l \in \mathscr{F}, p \in \mathscr{X}, t \in 2 . .|\mathscr{T}|, s \in \mathscr{S}
\end{aligned}
$$

Constraint (2) expresses the initial stocks of raw materials and goods present in industrial plants and distribution centers. Constraints (3) and (4) describe the storage of raw materials and finished products. Quantities must consider the inventory safety levels and must not exceed the storage capacity limits of each location. Constraints (5) and (6) mean that the number of lots of raw materials purchased must respect their availability with suppliers in each period. 
Table 1 - Deterministic and stochastic parameters of the 2SSP model.

\begin{tabular}{|c|c|c|}
\hline \multicolumn{2}{|c|}{ Deterministic parameters } & \multirow{2}{*}{$\frac{\text { Unit }}{\text { Binary }}$} \\
\hline$T_{l r p}^{R}$ & Matrix of technical route of product $p$ on resource $r$ at location $l$ & \\
\hline$B_{p^{\prime} p}$ & Bill of materials $p^{\prime}$ required to produce a unit of product $p$ & Scalar \\
\hline$M_{l r p}^{C}$ & Time unit required to produce product $p$ on resource $r$ at location $l$ & $\mathrm{~h}$ \\
\hline$E_{l r t}^{F}$ & Resource efficiency $r$ at location $l$ in period $t$ & $\%$ \\
\hline$A_{t}^{H}$ & Available hours in each period $t$ & $\mathrm{~h}$ \\
\hline$A_{l r t}^{X}$ & Extra hours available on resource $r$ at location $l$ in period $t$ & $\mathrm{~h}$ \\
\hline$L_{l p}^{M}$ & Lot size of product $p$ at location $l$ & Scalar \\
\hline$P_{l r t}^{M}$ & Preventive maintenance of resource $r$ at location $l$ in period $t$ & $\mathrm{~h}$ \\
\hline$S_{l p t}^{S}$ & Safety stock of product $p$ at location $l$ in period $t$ & Scalar \\
\hline$S_{l p t}^{X}$ & Stock capacity of product $p$ at location $l$ in period $t$ & Scalar \\
\hline$A_{l p t}^{R}$ & Availability of raw materials for product $p$ at location $l$ in period $t$ & Scalar \\
\hline$S_{l p}^{0}$ & Initial inventory of product $p$ at location $l$ & Scalar \\
\hline$Y_{l r}$ & Raw material yield on resource $r$ at location $l$ & $\%$ \\
\hline$N_{l r}^{M}$ & Number of resources of type $r$ at location $l$ & Scalar \\
\hline$T_{m l l^{\prime}}^{C X}$ & Transportation capacity of raw-material on modal $m$ from location $l$ to $l^{\prime}$ & Scalar \\
\hline$T_{m l l^{\prime}}^{C Y}$ & Transportation capacity of products on modal $m$ from location $l$ to $l^{\prime}$ & Scalar \\
\hline$C_{l t}^{I}$ & Inbound handling capacity at location $l$ in period $t$ & Scalar \\
\hline$C_{l t}^{O}$ & Outbound handling capacity on location $l$ and time period $t$ & Scalar \\
\hline$A_{l r t}^{V}$ & $\left(A_{t}^{H} N_{l r}^{M}-P_{l r t}^{M}\right)\left(E_{l r t}^{F} Y_{l r}\right)$ Availability of resource $r$ at location $l$ in period $t$ & $\mathrm{~h}$ \\
\hline \multicolumn{3}{|c|}{ Stochastic parameters } \\
\hline$D_{\text {cpts }}$ & Demand of customer $c$ for product $p$ in period $t$ and scenario $s$ & \\
\hline$R_{p s}$ & Sales revenue of finished product $p$ in scenario $s$ & \\
\hline$N_{p s}$ & Fictitious cost penalty for not meeting demand $p$ in scenario $s$ & \\
\hline$C_{l r s}^{F}$ & Fixed cost of resource $r$ at location $l$ in scenario $s$ & \\
\hline$C_{l p s}^{V}$ & Variable cost of production of $p$ at location $l$ in scenario $s$ & \\
\hline$C_{l r s}^{X}$ & Extra capacity cost on resource $r$ at location $l$ in scenario $s$ & \\
\hline$C_{l p s}^{S}$ & Unit inventory cost of product $p$ at location $l$ in scenario $s$ & \\
\hline$C_{m l l^{\prime} s}^{L}$ & Unit transport cost on modal $m$ from location $l$ to $l^{\prime}$ in scenario $s$ & \\
\hline$C_{l p s}^{P}$ & Unit procurement cost of raw material $x$ at location $l$ in scenario $s$ & \\
\hline$T_{l p s}^{X}$ & Tax over finished product $y$ sold to customer $c$ in scenario $s$ & \\
\hline$\rho_{s}$ & Probability of each scenario $s, \sum_{s \in \mathscr{S}} \rho_{s}=1$ & \\
\hline
\end{tabular}


Table 2 - First-stage and second-stage decision variables of the 2SSP model.

\begin{tabular}{ll}
\hline \multicolumn{2}{l}{ First-stage decision variables } \\
\hline$\alpha_{l p}$ & $\in \mathbb{Z}^{+}:$Multiple of lot-size for production of product $p$ at location $l$ in the first period \\
$a_{l r p}$ & Production of product $p$ on resource $r$ at location $l$ in the first period \\
$b_{l p}$ & Consumption of raw material $p$ at location $l$ in the first period \\
$s_{l p t}$ & Stock of product $p$ at location $l$ at the end of the first period \\
$d_{l p}$ & Met demand of product $p$ at location $l$ in the first period \\
$n_{l p 1 s}$ & Non-satisfied demand of product $p$ at location $l$ in the first period and scenario $s$ \\
$r_{l p}$ & $\in \mathbb{Z}^{+}:$Multiple of lot-size for procurement of raw material $p$ at location $l$ in the first period \\
$t_{m l l^{\prime} p}$ & Transportation of product $p$ on modal $m$ from $l$ to $l^{\prime}$ in the first period \\
$c_{l r}$ & Consumption of resource $r$ at location $l$ in the first period \\
$c_{l r}^{\prime}$ & Overtime percentage for resource $r$ at location $l$ in the first period \\
$y_{l r}$ & $\in\{0,1\}:$ Activate (or not) resource $r$ at location $l$ in the first period \\
\hline
\end{tabular}

Second-stage decision variables

$\alpha_{l p t s} \in \mathbb{Z}^{+}$: Production of product $p$ at location $l$ in period $t$ and scenario $s$

$a_{\text {lrpts }} \quad$ Production of product $p$ on resource $r$ at location $l$ in period $t$ and scenario $s$

$b_{l p t s} \quad$ Consumption of raw material $p$ at location $l$ in period $t$ and scenario $s$

$s_{l p t s} \quad$ Stock of product $p$ at location $l$ at end of period $t$ in scenario $s$

$d_{l p t s} \quad$ Met demand of product $p$ at location $l$ in period $t$ and scenario $s$

$n_{l p t s} \quad$ Non-satisfied demand of product $p$ at location $l$ in period $t$ and scenario $s$

$r_{\text {lpts }} \in \mathbb{Z}^{+}$: Procurement of raw material $p$ at location $l$ in period $t$ and scenario $s$

$t_{m l l^{\prime} p t s} \quad$ Transportation of product $p$ on modal $m$ from $l$ to $l^{\prime}$ in period $t$ and scenario $s$

$c_{l r t s} \quad$ Consumption of resource $r$ at location $l$ in period $t$ and scenario $s$

$c_{l r t s}^{\prime} \quad$ Overtime percentage for resource $r$ at location $l$ in period $t$ and scenario $s$

$y_{\text {lrts }} \in\{0,1\}$ : Activate (or not) resource $r$ at location $l$ in period $t$ and scenario $s$ 
Table 3 - Objective function elements of four-echelon SC planning model.

\begin{tabular}{ll}
\hline Elements $^{\mathbf{a}}$ & Description \\
\hline$R_{1}^{P}$ & After-tax revenue from sales in the first-stage \\
$C_{1}^{L}$ & Logistics cost on different transport modes in the first-stage \\
$C_{1}^{F}$ & Fixed cost for machine activation in each plant in the first-stage \\
$C_{1}^{V}$ & Finished product cost in each plant in the first-stage \\
$C_{1}^{P}$ & Procurement cost of raw material in the first-stage \\
$C_{1}^{S}$ & Storage cost in each plant in the first-stage \\
$C_{1}^{X}$ & Capacity expansion cost of resources in each plant in the first-stage \\
$C_{1}^{N}$ & Non-delivery cost for each customer in the first-stage \\
$R_{2}^{P}$ & Revenue after taxes from sales in the second-stage \\
$C_{2}^{L}$ & Logistics cost on different transport modes in the second-stage \\
$C_{2}^{F}$ & Fixed cost for machine activation in each plant in the second-stage \\
$C_{2}^{V}$ & Finished product cost in each plant in the second-stage \\
$C_{2}^{P}$ & Procurement cost of raw material in the second-stage \\
$C_{2}^{S}$ & Storage cost in each plant in the second-stage \\
$C_{2}^{X}$ & Capacity expansion cost of resources in each plant in the second-stage \\
$C_{2}^{N}$ & Non-delivery cost for each customer in the second-stage \\
\hline
\end{tabular}

${ }^{a}$ Elements subscript 1 and 2 represents the first-stage ${ }_{[1]}$ and the second-stage ${ }_{[2]}$.

$$
\begin{aligned}
& R_{1}^{P}=\sum_{l \in \mathscr{C}} \sum_{p \in \mathscr{Y}}\left(R_{p}-T_{l p}^{X}\right) d_{l p} \quad C_{1}^{L}=\sum_{m \in \mathscr{M}} \sum_{l l^{\prime} \in \mathscr{K}} \sum_{p \in \mathscr{Y}} C_{m l l^{\prime} s}^{L} t_{m l l^{\prime} p} \\
& C_{1}^{F}=\sum_{l \in \mathscr{I}} \sum_{r \in \mathscr{R}} C_{l r s}^{F} y_{l r} \quad C_{1}^{V}=\sum_{l \in \mathscr{I}} \sum_{p \in \mathscr{Y}} C_{l p s}^{V} \alpha_{l p} \\
& C_{1}^{P}=\sum_{l \in \mathscr{F}} \sum_{p \in \mathscr{P}} C_{l p s}^{P} r_{l p} \quad C_{1}^{S}=\sum_{l \in \mathscr{I}} \sum_{p \in \mathscr{Y}} C_{l p s}^{S} s_{l p} \\
& C_{1}^{X}=\sum_{l \in \mathscr{I}} \sum_{r \in \mathscr{R}} C_{l r s}^{X} c_{l r}^{\prime} \quad C_{1}^{N}=\sum_{s \in \mathscr{S}} \sum_{l \in \mathscr{C}} \sum_{p \in \mathscr{Y}} N_{p s} n_{l p} \\
& R_{2}^{P}=\sum_{l \in \mathscr{C}} \sum_{p \in \mathscr{Y}} \sum_{t>1}\left(R_{p s}-T_{l p s}^{X}\right) d_{l p t s} \quad C_{2}^{F}=\sum_{l \in \mathscr{I}} \sum_{r \in \mathscr{R}} \sum_{t>1} C_{l r s}^{F} y_{l r t s} \\
& C_{2}^{L}=\sum_{m \in \mathscr{M}} \sum_{l l^{\prime} \in \mathscr{K}} \sum_{p \in \mathscr{Y}} \sum_{t>1} C_{m l l^{\prime} s}^{L} t_{m l l^{\prime} p t s} \quad C_{2}^{V}=\sum_{l \in \mathscr{I}} \sum_{p \in \mathscr{Y}} \sum_{t>1} C_{l p s}^{V} \alpha_{l p t s} \\
& C_{2}^{P}=\sum_{l \in \mathscr{F}} \sum_{p \in \mathscr{P}} \sum_{t>1} C_{l p s}^{P} r_{l p t s} \quad C_{2}^{S}=\sum_{l \in \mathscr{I}} \sum_{p \in \mathscr{Y}} \sum_{t>1} C_{l p s}^{S} s_{l p t s} \\
& C_{2}^{X}=\sum_{l \in \mathscr{I}} \sum_{r \in \mathscr{R}} \sum_{t>1} C_{l r s}^{X} c_{l r t s}^{\prime} \quad C_{2}^{N}=\sum_{s \in \mathscr{S}} \sum_{l \in \mathscr{C}} \sum_{p \in \mathscr{Y}} N_{p s} n_{l p t s}
\end{aligned}
$$




$$
\begin{aligned}
& L_{l p}^{M} r_{l p}=\sum_{m \in \mathscr{M}} \sum_{l l^{\prime} \in \mathscr{K}} t_{m l l^{\prime} p} \\
& \forall l \in \mathscr{F}, p \in \mathscr{X}, t=1 \\
& L_{l p}^{M} r_{l p t s}=\sum_{m \in \mathscr{M}} \sum_{l l^{\prime} \in \mathscr{K}} t_{m l l^{\prime} p t s} \\
& \forall l \in \mathscr{F}, p \in \mathscr{X}, t \in 2 . .|\mathscr{T}|, s \in \mathscr{S} \\
& \forall l \in \mathscr{I}, p \in \mathscr{X}, t=1 \\
& \forall l \in \mathscr{I}, p \in \mathscr{X}, t \in 2 . .|\mathscr{T}|, s \in \mathscr{S} \\
& \sum_{m \in \mathscr{M}} \sum_{l^{\prime} l \in \mathscr{K}} t_{m l^{\prime} l p t s}+s_{l p t-1 s}=s_{l p t s}+b_{l p t s} \\
& L_{l p}^{M} \alpha_{l p}+\sum_{m \in \mathscr{M}} \sum_{l^{\prime} l \in \mathscr{K}} t_{m l^{\prime} l p}+S_{l p}^{0}= \\
& \sum_{m \in \mathscr{M}} \sum_{l l^{\prime} \in \mathscr{K}} t_{m l l^{\prime} p}+s_{l p t} \\
& L_{l p}^{M} \alpha_{l p t s}+\sum_{m \in \mathscr{M}} \sum_{l^{\prime} l \in \mathscr{K}} t_{m l^{\prime} l p t s}+s_{l p t-1 s}= \\
& \sum_{m \in \mathscr{M}} \sum_{l l^{\prime} \in \mathscr{K}} t_{m l l^{\prime} p t s}+s_{l p t s} \\
& \sum_{m \in \mathscr{M}} \sum_{l^{\prime} l \in \mathscr{K}} t_{m l^{\prime} l p}+S_{l p}^{0}=\sum_{m \in \mathscr{M}} \sum_{l l^{\prime} \in \mathscr{K}} t_{m l l^{\prime} p}+s_{l p t} \\
& \sum_{m \in \mathscr{M}} \sum_{l^{\prime} l \in \mathscr{K}} t_{m l^{\prime} l p t s}+s_{l p t-1 s}= \\
& \sum_{m \in \mathscr{M}} \sum_{l l^{\prime} \in \mathscr{K}} t_{m l l^{\prime} p t s}+s_{l p t s} \\
& \sum_{m \in \mathscr{M}} \sum_{l^{\prime} l \in \mathscr{K}} t_{m l^{\prime} l p}=d_{l p} \\
& \sum_{m \in \mathscr{M}} \sum_{l^{\prime} l \in \mathscr{K}} t_{m l^{\prime} l p t s}=d_{l p t s} \\
& \forall l \in \mathscr{I}, p \in \mathscr{Y}, t=1 \\
& \forall l \in \mathscr{I}, p \in \mathscr{Y}, t \in 2 . .|\mathscr{T}|, s \in \mathscr{S} \\
& \forall l \in \mathscr{H}, p \in \mathscr{Y}, t=1 \\
& \forall l \in \mathscr{H}, p \in \mathscr{Y}, t \in 2 . .|\mathscr{T}|, s \in \mathscr{S} \\
& \forall l \in \mathscr{C}, p \in \mathscr{Y}, t=1 \\
& \forall l \in \mathscr{C}, p \in \mathscr{Y}, t \in 2 . .|\mathscr{T}|, s \in \mathscr{S}
\end{aligned}
$$

The end of each period is connected by the sum of the input and output flows; consequently, the transportation of products is not permitted if the product does not reach the destination within the planned horizon. The input and output flows are respected for each location, product, and period. Equations (7) and (8) refer to the procurement and transportation of raw materials to industrial plants. Equations (9) and (10) represent the stock flow of raw material and its consumption for producing finished products. The input flow is expressed by the transport of raw materials or finished products from the preceding SC echelon, the production of lots of goods, the inventory level, and the procurement of multiple lots of raw materials the end of the previous period. Equations (7) to (10) represent the flow of raw materials, while equations (11) and (12) represent the flow of finished products in industrial plants. Equations (13) and (14) represent the flow of finished products on distribution centers, and equations (15) and (16) represent the transportation and delivery of finished products to customers. The output flow is the result of the balance of shipment of items to the subsequent SC echelon, the satisfied demand, the inventory level, and the consumption of raw materials in processes at the end of a period. 


$$
\begin{aligned}
& \sum_{m \in \mathscr{M}} \sum_{l^{\prime} l \in \mathscr{K}} \sum_{p \in \mathscr{Y}} t_{m l^{\prime} l p} \leq C_{l t}^{I} \\
& \sum_{m \in \mathscr{M}} \sum_{l^{\prime} l \in \mathscr{K}} \sum_{p \in \mathscr{Y}} t_{m l^{\prime} l p t s} \leq C_{l t}^{I} \\
& \sum_{m \in \mathscr{M}} \sum_{l l^{\prime} \in \mathscr{K}} \sum_{p \in \mathscr{Y}} t_{m l l^{\prime} p} \leq C_{l t}^{O} \\
& \sum_{m \in \mathscr{M}} \sum_{l l^{\prime} \in \mathscr{K}} \sum_{p \in \mathscr{Y}} t_{m l l^{\prime} p t s} \leq C_{l t}^{O} \\
& \sum_{p \in \mathscr{Y}} a_{l r p} M_{l r p}^{C}=c_{l r} \\
& \sum_{p \in \mathscr{Y}} a_{l r p t s} M_{l r p}^{C}=c_{l r t s} \\
& c_{l r} \leq A_{l r t}^{V} y_{l r}+c_{l r}^{\prime} A_{l r t}^{X} \\
& c_{l r t s} \leq A_{l r t}^{V} y_{l r t s}+c_{l r t}^{\prime} A_{l r t}^{X} \\
& \forall l \in \mathscr{H}, t=1 \\
& \forall l \in \mathscr{H}, t \in 2 . .|\mathscr{T}|, s \in \mathscr{S} \\
& \forall l \in \mathscr{H}, t=1 \\
& \forall l \in \mathscr{H}, t \in 2 . .|\mathscr{T}|, s \in \mathscr{S} \\
& \forall l \in \mathscr{I}, r \in \mathscr{R}, t=1 \\
& \forall l \in \mathscr{I}, r \in \mathscr{R}, t \in 2 . .|\mathscr{T}|, s \in \mathscr{S} \\
& \forall l \in \mathscr{I}, r \in \mathscr{R}, t=1 \\
& \forall l \in \mathscr{I}, r \in \mathscr{R}, t \in 2 . .|\mathscr{T}|, s \in \mathscr{S}
\end{aligned}
$$

Constraints (17) to (20) describe the inbound and outbound handling capacities at the distribution centers for each period. The production in each process depends on the route and production time of each item. Equations (21) and (22) represent the production capacity use. Constraints (23) and (24) express the capacity of a process, which is ruled by the available production time. In this period, a process may or may not be activated. If activated, its capacity can be reduced by implementing a preventive maintenance, for instance.

$$
\begin{aligned}
& c_{l p}^{\prime} \leq y_{l r} \\
& c_{l p t s}^{\prime} \leq y_{l r t s}
\end{aligned}
$$$$
\forall l \in \mathscr{I}, r \in \mathscr{R}, t=1
$$

Constraints (25) and (26) mean that the choice to overtime can be a profitable option. The use of extra capacity results in extra costs, which are included in the objective function. However, the value of the extra costs is bounded by the company. These constraints also ensure that extra capacity can be activated only if there is a requirement for production in the period 


$$
\begin{aligned}
& a_{l r p} T_{l r p}^{R}=L_{l p}^{M} \alpha_{l p} \\
& \forall l \in \mathscr{I}, r \in \mathscr{R}, p \in \mathscr{P}, t=1 \\
& a_{l r p t s} T_{l r p}^{R}=L_{l p}^{M} \alpha_{l p t s} \\
& \forall l \in \mathscr{I}, r \in \mathscr{R}, p \in \mathscr{P}, t \in 2 . .|\mathscr{T}|, s \in \mathscr{S} \\
& b_{l p^{\prime}}=\sum_{p \in \mathscr{Y}} B_{p^{\prime} p} L_{l p}^{M} \alpha_{l p} \\
& \forall l \in \mathscr{I}, p^{\prime} \in \mathscr{X}, t=1 \\
& b_{l p^{\prime} t s}=\sum_{p \in \mathscr{Y}} B_{p^{\prime} p} L_{l p}^{M} \alpha_{l p t s} \\
& \forall l \in \mathscr{I}, p^{\prime} \in \mathscr{X}, t \in 2 . .|\mathscr{T}|, s \in \mathscr{S} \\
& \sum_{p \in \mathscr{X}} t_{m l l^{\prime} p} \leq T_{m l l^{\prime}}^{C X} \\
& \sum_{p \in \mathscr{X}} t_{m l l^{\prime} p t s} \leq T_{m l l^{\prime}}^{C X} \\
& \sum_{p \in \mathscr{Y}} t_{m l l^{\prime} p} \leq T_{m l l^{\prime}}^{C Y} \\
& \sum_{p \in \mathscr{Y}} t_{m l l^{\prime} p t s} \leq T_{m l l^{\prime}}^{C Y} \\
& \forall m \in \mathscr{M}, l l^{\prime} \in \mathscr{K}, t=1 \\
& \forall m \in \mathscr{M}, l l^{\prime} \in \mathscr{K}, t \in 2 . .|\mathscr{T}|, s \in \mathscr{S} \\
& \forall m \in \mathscr{M}, l l^{\prime} \in \mathscr{K}, t=1 \\
& \forall m \in \mathscr{M}, l l^{\prime} \in \mathscr{K}, t \in 2 . .|\mathscr{T}|, s \in \mathscr{S} \\
& \forall l \in \mathscr{C}, p \in \mathscr{Y}, s \in \mathscr{S}, t=1 \\
& \forall l \in \mathscr{C}, p \in \mathscr{Y}, t \in 2 . .|\mathscr{T}|, s \in \mathscr{S} \\
& \forall l \in \mathscr{L}, p \in \mathscr{P}, t=1 \\
& \forall l \in \mathscr{L}, p \in \mathscr{P}, t \in 2 . .|\mathscr{T}|, s \in \mathscr{S} \\
& \forall m \in \mathscr{M}, l l^{\prime} \in \mathscr{L}, p \in \mathscr{P} \\
& \forall m \in \mathscr{M}, l l^{\prime} \in \mathscr{L}, p \in \mathscr{P}, t \in 2 . .|\mathscr{T}|, s \in \mathscr{S} \\
& \forall l \in \mathscr{L}, p \in \mathscr{P} \\
& \forall l \in \mathscr{L}, p \in \mathscr{P}, t \in 2 . .|\mathscr{T}|, s \in \mathscr{S} \\
& \forall l \in \mathscr{I}, r \in \mathscr{R}, p \in \mathscr{P} \\
& \forall l \in \mathscr{I}, r \in \mathscr{R}, p \in \mathscr{P}, t \in 2 . .|\mathscr{T}|, s \in \mathscr{S} \\
& \forall l \in \mathscr{I}, r \in \mathscr{R} \\
& \forall l \in \mathscr{I}, r \in \mathscr{R}, t \in 2 . .|\mathscr{T}|, s \in \mathscr{S} \\
& \forall l \in \mathscr{I}, r \in \mathscr{R} \\
& \forall l \in \mathscr{I}, r \in \mathscr{R}, t \in 2 . .|\mathscr{T}|, s \in \mathscr{S}
\end{aligned}
$$

Constraints (27) and (28) assure that a finished product is released by the latest machine of the product line routing in each plant. Constraints (29) and (30) express the bill of materials for a generic product structure (Pochet \& Wolsey, 2006); accordingly, a finished product is a result of the combination of raw materials in different proportions. Constraints (31) to (34) guarantee that the product flow does not surpass the transportation capacity for each transport mode. Constraints (35) and (36) indicate that eventually, part of the original demand may not be satisfied. Constraints (37) to (48) define the domain of the variables. 


\section{MULTI-CUT BENDERS DECOMPOSITION}

Stochastic programming problems take uncertainty into account. The problems tend to grow and require significant computational resource. This section proposes a relaxation followed by a multi-cut decomposition strategy to solve the stochastic original problem. The procedure consists in decomposing a complete deterministic equivalent problem into a Master Problem (MP) and relaxed Slave Problems (SP) where recourse decisions are taken. The optimization model with first-stage integer variables (on the first planning period) and second-stage continuous variables (on complementary planning periods) can be approached by the L-Shaped Method (Van Slyke \& Wets, 1969; Laporte \& Louveaux, 1993). The method is a scenario-based decomposition structure based on Benders decomposition (Benders, 1962) which is employed to stochastic optimization. The MP can be reformulated as follows:

$$
\max \Psi=R_{1}^{P}-C_{1}^{L}-C_{1}^{F}-C_{1}^{V}-C_{1}^{P}-C_{1}^{S}-C_{1}^{X}+\theta
$$

Subjected to first period $(t=1)$ constraints: (2) (3) (5) (7) (9) (11) (13) (15) (17) (19) (21) (23) (25) (27) (29) (31) (33) (35) (37) (39) (41) (43) (45) (47)

$$
\theta \leq \mathscr{Q}(\alpha, y, r)
$$

The variable $\theta$ introduced in the objective function (49) provides a connection between the MP and each scenario SP, however, since the proposed constraint (50) is not defined explicitly, it can not be used computationally as a constraint, so this constraint is replaced by a number of cuts, generated from dual vectors of SP, which are gradually added to the MP in an iterative process. The SPs are reformulated as follows:

$$
\max \Phi=\sum_{s \in \mathscr{S}} \rho_{s}\left(R_{2}^{P}-C_{2}^{L}-C_{2}^{F}-C_{2}^{V}-C_{2}^{P}-C_{2}^{S}-C_{2}^{X}\right)
$$

Subjected to the following periods $(t \in 2 . . \mid \mathscr{T})$ constraints: (4) (6) (8) (10) (12) (14) (16) (18) (20) (22) (24) (26) (28) (30) (32) (34) (36) (38) (40) (42) (44) (46) (48)

The proposed model with relaxed second-stage variables has complete recourse (Birge \& Louveaux, 2011); therefore, for any feasible first-stage solution, the second-stage problem is always feasible, so only optimality cuts are needed in the Benders stochastic decomposition. On a single-cut approach, the number of iterations needed for reaching the optimum grows exponentially with the number of realizations. The advantage of the proposed method is that for the multi-cut approach it grows linearly (Oliveira et al., 2014).

Let $n \in N$ be the index of iterations needed for reaching the optimum. In order to accelerate the BD algorithm, we decompose the variable $\theta$ for each scenario $s$ to return the number of cuts equivalent to the number of scenarios for each iteration $n$. We define $\pi_{i} \in \Pi$ as the optimal extreme point of the dual polyhedron $\Pi$ resulted from constraints $i=(4),(6),(10),(12),(14)$ (18), (20), (32), (34), and (36). However, we consider only a subset $\Pi^{\prime}$ of $\Pi$ because cuts are 
added iteratively. The Inequality (50) is replaced by optimality multi-cuts (52), that link the MP and SP scenarios.

$$
\begin{aligned}
& \theta_{s} \leq \sum_{l \in(\mathscr{I} \cup \mathscr{H})} \sum_{p \in \mathscr{P}} \sum_{t \in 2 . . \mid} \pi_{(4)} S_{l p t}^{S}+\sum_{l \in(\mathscr{I} \cup \mathscr{H})} \sum_{p \in \mathscr{P}} \sum_{t \in 2 . . \mid} \pi_{(4)} S_{l p t}^{X}+\sum_{l \in \mathscr{F}} \sum_{p \in \mathscr{P}} \sum_{t \in 2 . .|\mathscr{T}|} \pi_{(6)} A_{l p t}^{R} \\
& -\sum_{l \in \mathscr{I}} \sum_{p \in \mathscr{X}} \sum_{t \in 2 . .|\mathscr{T}|} \pi_{(10)} s_{l p t_{-1} s}-\sum_{l \in \mathscr{I}} \sum_{p \in \mathscr{Y}} \sum_{t \in 2 . . \mid} \pi_{(12)} s_{l p t_{-1} s}-\sum_{l \in \mathscr{H}} \sum_{p \in \mathscr{Y}} \sum_{t \in 2 . .|\mathscr{T}|} \pi_{(14)} s_{l p t_{-1} s} \\
& +\sum_{l \in \mathscr{H}} \sum_{t \in 2 . .|\mathscr{T}|} \pi_{(18)} C_{l t}^{I}+\sum_{l \in \mathscr{H}} \sum_{t \in 2 . .|\mathscr{T}|} \pi_{(20)} C_{l t}^{O}+\sum_{m \in \mathscr{M}} \sum_{l l^{\prime} \in \mathscr{K}} \sum_{t \in 2 . .|\mathscr{T}|} \pi_{(32)} T_{m l l^{\prime}}^{C X} \\
& +\sum_{m \in \mathscr{M}} \sum_{l l^{\prime} \in \mathscr{K}} \sum_{t \in 2 . . \mid} \pi_{(34)} T_{m l l^{\prime}}^{C Y}+\sum_{l \in \mathscr{C}} \sum_{p \in \mathscr{Y}} \sum_{t \in 2 . .|\mathscr{T}|} \pi_{(36)} D_{t p c s}, \quad \forall s \in \mathscr{S}, \pi_{i} \in \Pi^{\prime}
\end{aligned}
$$

The proposed Algorithm 1 is applied for solving of the mixed-integer 2SSP SC planning problem. It consists in relaxing the SP integrality constraints, conducting the multi-cut BD approach, and recovering the integrality constraints for a branch and bound or branch and cut scheme (Birge \& Louveaux, 2011) while it creates non-examined nodes. The strategy is evaluated by numerical experiments and a case study.

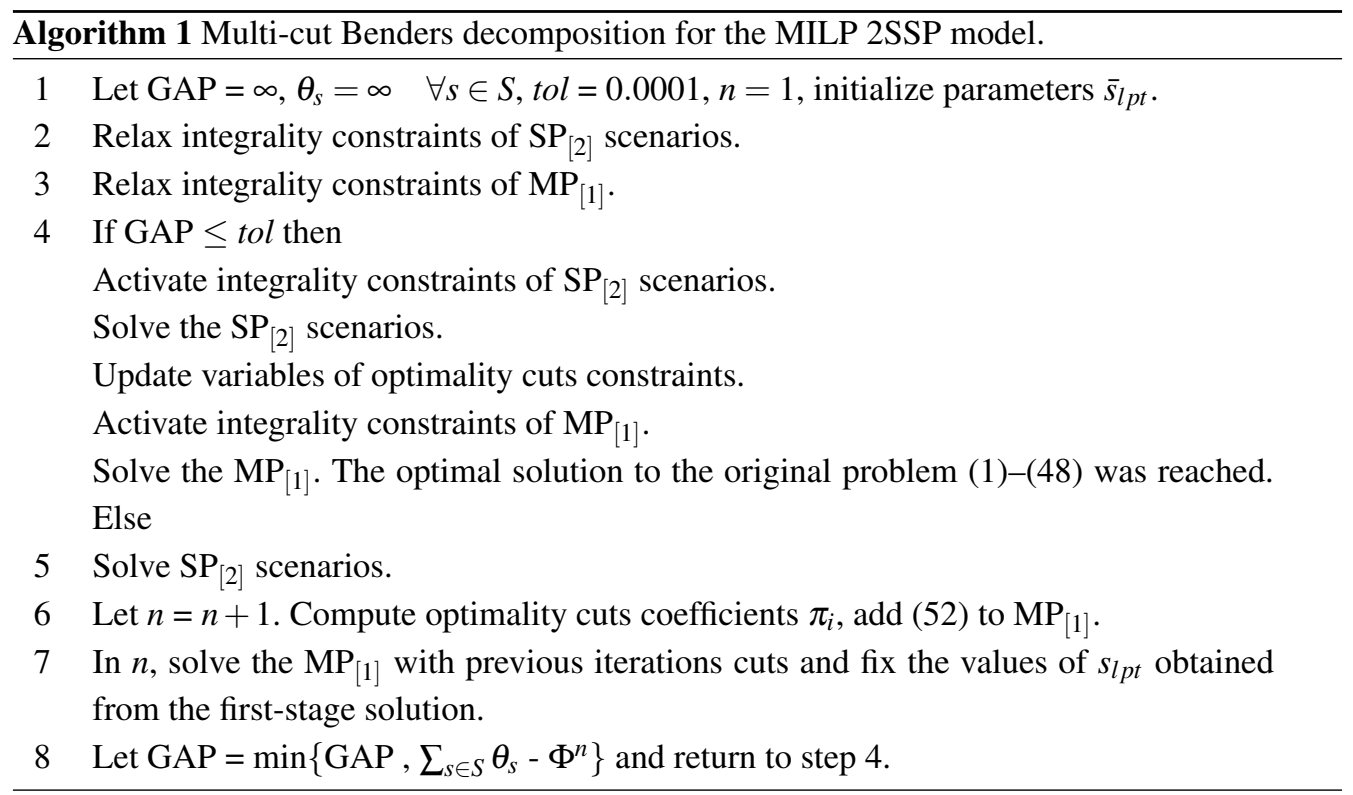

Elements subscript 1 and 2 represents first stage ${ }_{[1]}$ and second stage $[2]$.

\section{NUMERICAL EXPERIMENTS}

In this section numerical experiments are conducted to evaluate the computational performance of the proposed algorithm on solving a medium-sized 2SSP SC problem with 6 suppliers, 2 industrial plants, 4 distribution centers, 20 demand clusters, 8 types of raw materials, 10 production resources, 20 product families, and 2 modes of transport over a planning period of 12 months. The models were implemented in AMPL ${ }^{\mathrm{TM}}$ (Fourer et al., 2003) and solved with Gurobi $9.0^{\mathrm{TM}}$ in 
a Linux Mint 17.3 64-bit, RAM of $16 \mathrm{~GB}$, and an Intel Core I5 2.50 GHz. Gurobi used dual simplex LP optimizer with presolve activated, and branch-and-cut with simplex for MIP optimizer with cutting planes activated (Gomory, Implied bound, MIR, Flow cover, Zero half), and multi-thread (thread count was 4 of 4 available processors).

Table 4 - Size and solving time of 2SSP for LP and MILP Monolithic model (M) and Multi-Cut Benders Decomposition (MC Dec.) model.

\begin{tabular}{ccccccccc}
\hline & & & & & \multicolumn{2}{c}{ LP(CPU(s) } & \multicolumn{2}{c}{ MILP $(\mathbf{C P U}(\mathbf{s}))$} \\
\cline { 7 - 9 } Scen & Const. & Var. & Int. & Bin. & M & MC Dec. & M $^{\mathrm{a}}$ & MC Dec. \\
\hline $\mathbf{2 0}$ & 71,964 & 151,082 & 3,319 & 221 & 3.50 & 7.09 & 124.62 & 56.49 \\
$\mathbf{4 0}$ & 143,704 & 301,582 & 6,619 & 441 & 8.95 & 14.95 & $1,131.98$ & 117.86 \\
$\mathbf{6 0}$ & 215,444 & 452,082 & 9,919 & 661 & 14.31 & 22.82 & $3,373.13$ & 341.52 \\
$\mathbf{8 0}$ & 287,184 & 602,582 & 13,219 & 881 & 21.72 & 30.72 & $5,609.30$ & 524.31 \\
$\mathbf{1 0 0}$ & 358,924 & 753,082 & 16,519 & 1,101 & 28.39 & 39.75 & $10,000.00^{*}$ & 591.61 \\
$\mathbf{1 2 0}$ & 430,664 & 903,582 & 19,819 & 1,321 & 39.19 & 48.09 & $* *$ & 788.24 \\
$\mathbf{1 4 0}$ & 502,404 & $1,054,082$ & 23,119 & 1,541 & 78.35 & 57.47 & $* *$ & 874.88 \\
$\mathbf{1 6 0}$ & 574,144 & $1,204,582$ & 26,419 & 1,761 & 153.19 & 65.95 & $* *$ & $1,216.40$ \\
$\mathbf{1 8 0}$ & 645,884 & $1,355,082$ & 29,719 & 1,981 & 175.58 & 77.19 & $* *$ & $1,796.82$ \\
$\mathbf{2 0 0}$ & 717,624 & $1,505,582$ & 33,019 & 2,201 & 306.37 & 94.25 & $* *$ & $3,547.75$ \\
\hline a*No solution. ${ }^{* *}$ No RAM. & & & & & & &
\end{tabular}

Table 5 - Statistical analysis carried out on different scenarios sets.

\begin{tabular}{lllllllllll}
\hline Scen. & $\mathbf{2 0}$ & $\mathbf{4 0}$ & $\mathbf{6 0}$ & $\mathbf{8 0}$ & $\mathbf{1 0 0}$ & $\mathbf{1 2 0}$ & $\mathbf{1 4 0}$ & $\mathbf{1 6 0}$ & $\mathbf{1 8 0}$ & $\mathbf{2 0 0}$ \\
\hline Min. & 8,272 & 7,329 & 7,932 & 7,487 & 7,660 & 7,438 & 7,569 & 7,132 & 7,461 & 7,255 \\
$\mathrm{Q} 1$ & 8,798 & 8,725 & 8,537 & 8,335 & 8,470 & 8,650 & 8,344 & 8,303 & 8,456 & 8,327 \\
$\mathrm{Q} 2$ & 9,130 & 8,964 & 8,860 & 8,759 & 8,756 & 9,027 & 8,805 & 8,720 & 8,873 & 8,717 \\
$\bar{X}$ & 9,198 & 8,952 & 8,858 & 8,768 & 8,804 & 8,994 & 8,756 & 8,710 & 8,829 & 8,702 \\
$\sigma$ & 490 & 526 & 505 & 585 & 554 & 558 & 576 & 602 & 537 & 549 \\
$\sigma_{\bar{X}}$ & 109 & 83 & 65 & 65 & 55 & 51 & 49 & 48 & 40 & 39 \\
Q3 & 9,436 & 9,312 & 9,137 & 9,148 & 9,173 & 9,443 & 9,130 & 9,119 & 9,133 & 9,046 \\
Max. & 10,262 & 9,883 & 10,097 & 10,084 & 9,998 & 10,100 & 10,143 & 10,342 & 10,340 & 10,406 \\
C.I. $(95 \%)$ & 215 & 133 & 128 & 128 & 109 & 100 & 95 & 93 & 79 & 76 \\
\hline
\end{tabular}

The experiment consists on solving 10 test-problems with a number of scenarios ranging from 20 to 200 in increments of 20. The experiment comprises different instances of independent samples with random variables adopting the same parameters for all instances. The probability is uniformly distributed according to the number of scenarios. The optimization experiments run for up to 10,000 seconds to evaluate the efficiency of the proposed method compared to the monolithic model.

Table 4 presents the problems size and shows the effect of the multi-cut BD algorithm applied to the relaxed LP and to the MILP version of the 2SSP model, and Table 5 presents the statistical analysis of the scenarios outputs. The results illustrated in Figure 1 suggest that the expected 


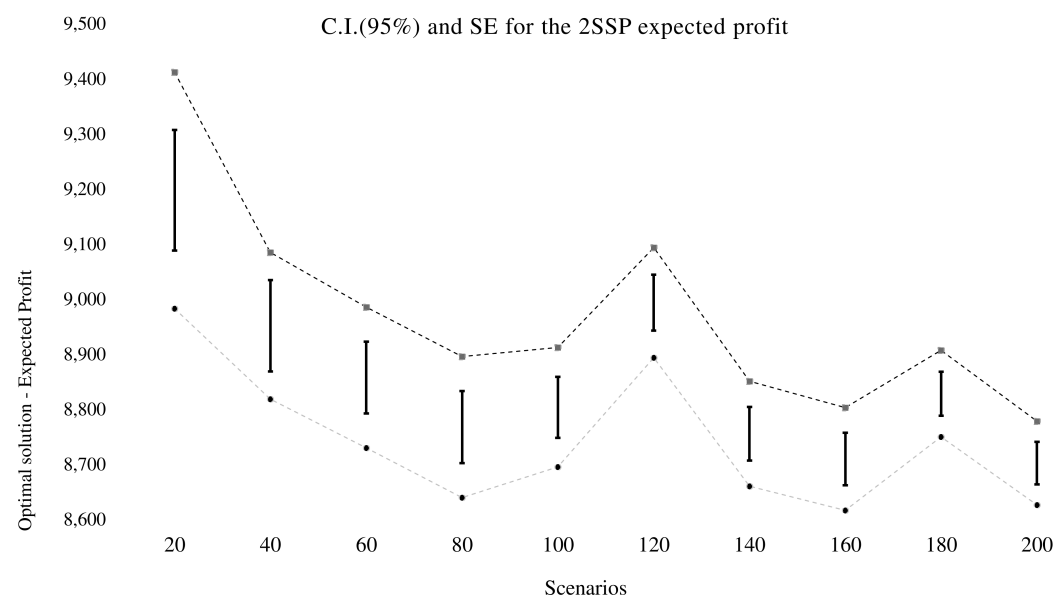

Figure 1 - The expected profit precision is increased in a rising number of scenarios. Confidence Interval (95\%) and Standard Error of the expected profit of 2SSP model.

profit precision is increased in a rising number of scenarios. The Figure 1 also shows the range of confidence interval of the expected profit and its standard error. The confidence interval and standard error are reduced and the number of scenarios is increased. The proposed multi-cut BD method is efficient on solving both linear and particularly mixed-integer problems. Decomposed 2SSP MILP problems take approximately one-tenth of the monolithic model solving time on instances with 20-80 scenarios and less than an hour for instances with 100-200 scenarios, which were not solved by monolithic model due to the overflow limit of computer memory. The method becomes more attractive as the instances increase in size. Although the results suggest the advantage of stochastic multi-cut BD method over the monolithic model, these results may not occur at all possible test-problems, nevertheless, they illustrate the potential of the proposed method. The multi-cut BD is efficient because when multiple cuts are applied in the MP, the number of iterations is significantly reduced, so the MP is solved in a short time, despite its large size.

\section{CASE STUDY}

The 2SSP model is applied to the tactical SC plan of a Brazilian flat steel chain that faces demand and price uncertainty. Over the last decade, the Organization for Economic Cooperation and Development (oe.cd/steelcapacity) revealed the steel over-capacity with structural supply-demand imbalances as a challenge to the global steel industry (Otsuka, 2017). Therefore, this flat steel industry redesigned its SC acquiring upstream mining operations and downstream distribution centers to hedge against price and demand variations over the long-term. In the medium term, this steel industry adopted the S\&OP methodology to balance demand and supply. The integration of medium-term tactical SC plan to short-term operational plan occurs through monthly review, following the S\&OP methodology to which the proposed 2SSP SC model is aligned. 
The integrated production and logistics process begins with the provision of ore and coal by three suppliers to two industrial plants, where they are converted to steel and transformed into 30 product families. Ships supply coal in multiple of 60,000, 120,000, and 150,000 tons, respectively, and the trains comprise 170 to 320 wagons, setting lots multiple of 17,000 tons and 32,000 tons. Industrial plants 1 and 2 contain 24 and 22 transformation processes, respectively. BOF furnaces size varies from 180 to 240 tons of steel, setting a batch production. The SC includes a complex logistics network with transshipment hubs at two ports, six distribution centers, and three transportation modes. The finished products, like slabs, plates, and coils, are shipped to 34 demand regions encompassing internal and external customers by railway, highway, or waterway over a planning horizon of 12 months.

The transport costs consist of average rates of cargo trucks, wagons or ships. Although the railway transport capacity is limited, third-party logistics (3PL) systems can expand the road transportation capacity. Processes analysts provided equipment capacity, product line routing, and production costs and time. The values of demand and price match the normal distribution, with average values derived from sales forecast and variance from sales histories. The normal distribution captures the essential characteristics of uncertainty and is often adopted in the literature (Gupta \& Maranas, 2003; You \& Grossmann, 2013).

Table 6 - Financial and operating results of the case study

\begin{tabular}{|c|c|c|c|}
\hline Financial report & Value (\$) & Operational report & Value (t.) \\
\hline Sales revenue & $1,910,739,797.99$ & Production on plant-[1] & $2,979,420$ \\
\hline Logistics cost & $511,717,155.55$ & Production on plant-[2] & $1,507,900$ \\
\hline Production fixed-cost & $5,987,185.18$ & Overtime on plant-[1](h) & $3,593.52$ \\
\hline Production variable-cost & $7,780,054.66$ & Overtime on plant-[2](h) & $2,241.92$ \\
\hline Procurement cost & $439,192,253.11$ & Transport on modal-[1] & $7,557,859$ \\
\hline Overtime cost & $18,677.87$ & Transport on modal-[2] & $5,245,359$ \\
\hline Inventory cost & $9,903,492.29$ & Transport on modal-[3] & $5,242,080$ \\
\hline Expected overall profit & $936,140,979.30$ & & \\
\hline Profit scenario-[1] & $964,289,708.25$ & Total demand & $4,929,647$ \\
\hline Profit scenario-[2] & $917,697,569.37$ & Satisfied demand & $4,609,799$ \\
\hline Profit scenario-[3] & $926,435,660.28$ & Unsatisfied demand & 319,848 \\
\hline \multicolumn{4}{|l|}{ Model statistics } \\
\hline Scenarios & 3 & Inventory on plant-[1] & $2,527,276$ \\
\hline Variables & 1.062 .079 & Inventory on plant-[2] & $2,436,179$ \\
\hline Integers & 6.936 & Inventory on DCs & $1,695,308$ \\
\hline Binaries & 1.632 & Inventory on Ports & 636,521 \\
\hline Constraints & 1.085 .067 & Ore procurement-[S1] & $4,320,000$ \\
\hline Time CPU(s) & $10.000,00$ & Ore procurement-[S2] & $2,080,000$ \\
\hline $\operatorname{Gap}(\%)$ & 0,97 & Coal procurement-[S3] & $3,150,000$ \\
\hline
\end{tabular}


The results of the 2SSP SC planning problem are presented in Table 6. For confidentiality reasons and respect for the company, the original data have been preserved. The demand was generated by a random procedure following the normal distribution with proportional data to validate the functionality to which the model is proposed. The financial-operational report presents the result of three random scenario planning. In these scenarios, the demand is lower than the nominal capacity. Production is concentrated in plant 1 where fixed and variable costs are lower. Some resources of plant 1 and plant 2 are used to maximum capacity, requiring expansion through overtime. It is justified when the product mix is heterogeneous. Still, $6.5 \%$ of the total demand is not satisfied. This occurs when products have high operating costs and do not share resources with other products line routes. In such occasions, the most profitable decision may be to disable a resource and lose sales.

For this scenario, the global demand is less than the plant nominal capacity, so the dominant strategy is to use plant 1 at maximum capacity, due to lower fixed and variable costs. Inventories are not fully used in the last month, due to safety stock constraints. On ports, the flow level is higher, since these transshipment hubs concentrate all foreign market demand.

The computational performance of the BD method applied to the case study model is also evaluated. Since the S\&OP process presumes the interactions of participants and, eventually, many optimizations run to obtain a general agreement for the tactical SC plan, the experiments considered a limit of 3,600 seconds for both decomposed and monolithic models. Results are presented in Table 7. The optimal solution of this 2SSP SC problem instances are difficult to obtain. The monolithic model did not find a feasible initial solution in experiments with three or more scenarios. However, the BD algorithm found solutions under an acceptable gap for problems with more than two million variables and constraints within one hour.

Table 7 - Performance of the 2SSP model for 1 - 6 scenarios.

\begin{tabular}{ccccccc}
\hline $\mathbf{S}$ & Constraints & Variables & Integer & Binary & Monolithic $^{\mathbf{a}}$ & Decomposed $^{\mathbf{b}}$ \\
\hline $\mathbf{1}$ & 382.487 & 374.343 & 2.448 & 576 & $\mathbf{0 , 7 6 \%}$ & $1,60 \%$ \\
$\mathbf{2}$ & 733.762 & 718.211 & 4.692 & 1.104 & $\mathbf{0 , 9 1 \%}$ & $1,14 \%$ \\
$\mathbf{3}$ & 1.085 .067 & 1.062 .079 & 6.936 & 1.632 & $* * * \%$ & $\mathbf{1 , 7 2 \%}$ \\
$\mathbf{4}$ & 1.436 .372 & 1.405 .947 & 9.180 & 2.160 & $* * * \%$ & $\mathbf{1 , 5 7 \%}$ \\
$\mathbf{5}$ & 1.787 .677 & 1.749 .815 & 11.424 & 2.688 & $* * * \%$ & $\mathbf{3 , 8 1 \%}$ \\
$\mathbf{6}$ & 2.138 .982 & 2.093 .683 & 13.668 & 3.216 & $*, * * \%$ & $\mathbf{1 , 5 3 \%}$ \\
\hline
\end{tabular}

${ }^{\mathrm{a}, \mathrm{b}}$ Optimality gap for 3,600 seconds * No initial solution.

Decision-makers often evaluate plans classified into pessimistic, most likely, and optimistic in corporate environments. Therefore, we set three random scenarios to compare the stochastic to the deterministic plan and to evaluate the case study' metrics of EVPI and VSS by the 2SSP SC model. The decomposed 2SSP model was run for 5,990.69 s until the solver reach optimal solution. The result is available in Table 8 . 
The EVPI is the expected value of perfect information, and consists in the difference between the average of the optimal solutions of the deterministic problem with the perfect information for each scenario and the solution of the stochastic programming model. The VSS is the value of the stochastic solution, and represents the difference between the result of the stochastic model, which adopts random parameters represented by a probability distribution, and the result of the deterministic model when adopting average values. Therefore, EVPI and VSS represent, respectively, the loss of profit in the presence of uncertainty and the likely gain on solving the stochastic model. The EVPI of this 2SSP SC case study is $\$ 233,370,620.63$. However, as the perfect information of all the planning periods is not available, this is only a hypothetical reference value. On the other hand, the VSS of the case study is $\$ 186,998,433.19$ revealing the superior quality of the stochastic model towards the deterministic model.

Table 8 - Case study EVPI and VSS analysis.

\begin{tabular}{lrlr}
\hline EVPI Analysis & Value (\$) & VSS Analysis & Value (\$) \\
\hline Profit scenario-[1] & $1,169,131,282.44$ & Profit $^{2}$ & $749,142,546.11$ \\
Profit scenario-[2] & $1,170,201,701.33$ & & \\
Profit scenario-[3] & $1,169,201,815.94$ & & \\
\hline
\end{tabular}

$\begin{array}{llll}\left(\mathbf{A}_{1}\right) \text { Expected profit } & 1,169,511,599.93 & \left(\mathbf{A}_{2}\right) \text { Expected profit } & 749,142,546.11\end{array}$

\section{SSP model}

\begin{tabular}{ll}
\hline Profit scenario-[1] & $964,289,708.25$ \\
Profit scenario-[2] & $917,697,569.37$ \\
Profit scenario-[3] & $926,435,660.28$ \\
\hline (B) Expected profit & $936,140,979.30$ \\
\hline
\end{tabular}

$\begin{array}{llll}\text { EVPI }\left(\mathbf{A}_{1}-\mathbf{B}\right): & 233,370,620.63 & \text { VSS }\left(\mathbf{B}-\mathbf{A}_{2}\right): & 186,998,433.19\end{array}$

${ }^{1}$ Deterministic instances with perfect information. ${ }^{2}$ Adopts average information.

Finally, we investigated the effect of using the 2SSP model for SC planning in an S\&OP context, where elements of SC may vary and impact the tactical plan. The experiment consists in changing proportionately the random parameters of the cost of raw materials, the price of the finished product and demand across $-20 \%,-10 \%, 10 \%$ to $20 \%$ and comparing to the baseline scenario the following performance indicators: expected profit, satisfied demand, unsatisfied demand and the SC inventory level.

The results suggest that the price reduction of ore and coal increases proportionally the overall SC profit. Nonetheless, the rise in raw materials price may cause stock disruption, reduce service level by increasing unsatisfied demand, and affect the overall SC profit. The price variation of the finished product, however, has a greater impact on overall profit. The Figure 2(b) illustrates the devastating effect to the company's results influenced by the products price reduction. 


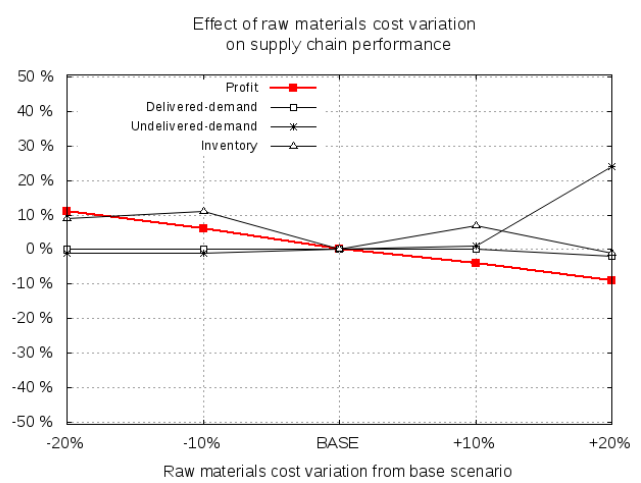

(a) Effect of raw materials cost variation.

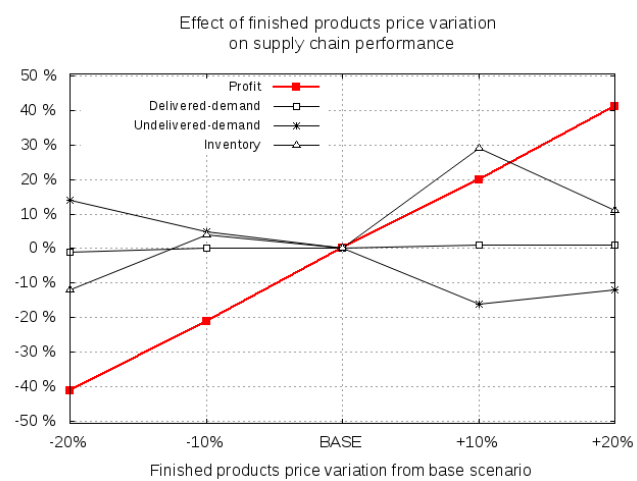

(b) Effect of product price variation.

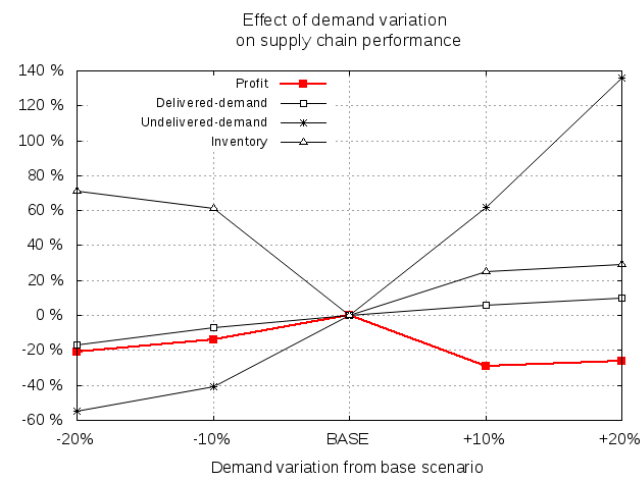

(c) Effect of demand variation.

Figure 2 - Operational and financial effect of the change in the raw materials costs and products prices.

This reduction can occur by sales discounts and by macroeconomic policy restrictions. On the other hand, policies that add value to the finished product and result in price increase, impact positively the overall SC profit. However, this increase in profit is also dependent on increasing the overall SC inventory levels. This result suggests that the reduction of unsatisfied demand is obtained by accumulation of finished products during periods of availability of production capacity.

The Figure 2(c) presents a counter-intuitive result. In this simulation, a demand variation reduces profit compared to the baseline scenario. This happens, for example, when the company adopts a strategy of increasing its market-share, but it has no power to influence the demand, adopting a reactive approach. In this case, the strategy can lead to an increase of heterogeneous demand resulting in lost sales. On the other hand, if a company has the power to influence demand, its strategy can lead to an increase of demand of products that could be allocated to resources with idle capacity. Thus, we conclude that the optimal SC profit is not proportional to demand. In these cases, the proactive attitude of the sales team contributes when it acts cohesively with the 
production, distribution and procurement team promoting the increase of demand of the ideal production mix.

\section{CONCLUSION}

This study proposed a multi-cut BD algorithm to solve a 2SSP model for the tactical SC planning admitting uncertainty and reflecting the rolling horizon planning practice in the context of S\&OP methodology. The algorithm and model were evaluated by numerical experiments and by a case study of a Brazilian flat steel industry that adopts the S\&OP for balancing supply and demand in the medium term.

Numerical experiments showed that the multi-cut BD method becomes more attractive as the problem increase in size. The proposed method solved large-scale instances taking nearly onetenth of the monolithic model solving time. The case study showed the 2SSP model's adequacy to the rolling horizon planning framework adopted on the S\&OP methodology. Successfully implementation, however, comes with top management support, cross-functional integration, metrics monitoring, appropriate information system, and training.

This study fills some literature gaps as the general model adequately tackles the S\&OP peculiar aspect of rolling horizon planning and the proposed multi-cut BD algorithm solve large-scale 2SSP MILP SC problems. In general, the findings suggest that the optimal SC profit is not proportional to demand due to capacity constraints along with the SC. Such findings reinforce the usefulness of the proposed model to support the S\&OP process raising the synergy of the sales team with the production, distribution, and procurement team.

Some limitations of the study are worth mentioning. Although numerical experiments considered up to 200 scenarios, the case study examined up to six situations due to RAM limitations. Therefore, the case study should consider a set of at least 30 scenarios to obtain better statistical significance to the expected value of the objective function. Further research may improve the multi-cut decomposition method and formulation with acceleration techniques, and the stochastic formulation admitting more elements of uncertainty. Additional avenues for developments include formulating capacity planning problem and multi-commodity network flow via nonlinear global optimization (Ferreira et al., 2013), or robust optimization models (Babazadeh \& Sabbaghnia, 2018) for examining multiple planning scenarios and alternative risk profiles. These procedures can increase the integration of executive leaders with the S\&OP team for a strategic S\&OP process.

\section{Acknowledgments}

The authors would like to thank the editor and referees for the comments and feedback that improved the quality of this paper. 


\section{References}

[1] Alem D \& Morabito R. 2013. Risk-averse two-stage stochastic programs in furniture plants. OR spectrum, 35(4): 773-806.

[2] Almeida JFdF, Conceição SV, Pinto LR, de CAmargo RS \& JÚnior GdM. 2018. Flexibility evaluation of multiechelon supply chains. PloS one, 13(3): e0194050.

[3] BA BH, Prins C \& Prodhon C. 2018. A generic tactical planning model to supply a biorefinery with biomass. Pesquisa Operacional, 38: 1-30. Available at: http://www. scielo.br/scielo.php?script=sci_arttext\&pid=S0101-74382018000100001\&nrm=iso.

[4] BAbazadeh R \& SAbBaghnia A. 2018. Evaluating the performance of robust and stochastic programming approaches in a supply chain network design problem under uncertainty. International Journal of Advanced Operations Management, 10(1): 1-18.

[5] Benders JF. 1962. Partitioning procedures for solving mixed-variables programming problems. Numerische mathematik, 4(1): 238-252.

[6] Birge JR \& Louveaux F. 2011. Introduction to stochastic programming. Springer Science \& Business Media.

[7] BUXEY G. 2003. Strategy not tactics drives aggregate planning. International Journal of Production Economics, 85(3): 331-346.

[8] Calfa BA, Agarwal A, Bury SJ, Wassick JM \& Grossmann IE. 2015. DataDriven Simulation and Optimization Approaches To Incorporate Production Variability in Sales and Operations Planning. Industrial \& Engineering Chemistry Research, 54(29): 7261-7272.

[9] CARniato A \& CAMPONOGARA E. 2011. Integrated coal-mining operations planning: modeling and case study. International Journal of Coal Preparation and Utilization, 31(6): 299-334.

[10] Chen-Ritzo CH, Ervolina T, Harrison TP \& Gupta B. 2010. Sales and operations planning in systems with order configuration uncertainty. European journal of operational research, 205(3): 604-614.

[11] Clark AJ \& SCARF H. 1960. Optimal policies for a multi-echelon inventory problem. Management science, 6(4): 475-490.

[12] Cunha P, Oliveira F \& Raupp FM. 2017. Periodic review system for inventory replenishment control for a two-echelon logistics network under demand uncertainty: A two-stage stochastic programing approach. Pesquisa Operacional, 37(2): 247-276.

[13] DantZig GB. 1955. Linear programming under uncertainty. Management science, 1(34): 197-206. 
[14] Dillon M, Oliveira F \& ABbasi B. 2017. A two-stage stochastic programming model for inventory management in the blood supply chain. International Journal of Production Economics, 187: 27-41.

[15] DutTa G \& Fourer R. 2001. A survey of mathematical programming applications in integrated steel plants. Manufacturing \& Service Operations Management, 3(4): 387-400.

[16] FABIAN T. 1958. A linear programming model of integrated iron and steel production. Management Science, 4(4): 415-449.

[17] Ferreira RPM, Luna HPL, Mahey P \& Souza MCD. 2013. Global optimization of capacity expansion and flow assignment in multicommodity networks. Pesquisa Operacional, 33(2): 217-234.

[18] Fourer R, Gay DM \& Kernighan BW. 2003. AMPL. 2nd ed.. Thomson Books. 517 pp.

[19] GuPTA A \& MARANAS CD. 2003. Managing demand uncertainty in supply chain planning. Computers \& Chemical Engineering, 27(8): 1219-1227.

[20] HAX AC \& MEAL HC. 1973. Hierarchical integration of production planning and scheduling. Sloan School of Management, Available at: http://hdl.handle.net/1721.1/1868.

[21] Kayvanfar V, Husseini SM, Sajadieh MS \& Karimi B. 2018. A multi-echelon multi-product stochastic model to supply chain of small-and-medium enterprises in industrial clusters. Computers \& Industrial Engineering, 115: 69-79.

[22] King AJ \& Wallace SW. 2012. Modeling with stochastic programming. Springer Science \& Business Media.

[23] Laporte G \& Louveaux FV. 1993. The integer L-shaped method for stochastic integer programs with complete recourse. Operations research letters, 13(3): 133-142.

[24] Moraes LA \& FARIA LF. 2016. A stochastic programming approach to liquified natural gas planning. Pesquisa Operacional, 36: 151-165. Available at: http://www.scielo.br/ scielo.php?script=sci_arttext\&pid=S0101-74382016000100151\&nrm=iso.

[25] MT Thomé A, Soucasaux Sousa R \& do Carmo LF. 2014. Complexity as contingency in sales and operations planning. Industrial Management \& Data Systems, 114(5): 678-695.

[26] Nemati Y, Madhoshi M \& Ghadikolaei AS. 2017. The effect of Sales and Operations Planning (S\&OP) on supply chain's total performance: A case study in an Iranian dairy company. Computers \& Chemical Engineering, 104: 323-338.

[27] Oliveira F, Grossmann IE \& Hamacher S. 2014. Accelerating Benders stochastic decomposition for the optimization under uncertainty of the petroleum product supply chain. Computers \& Operations Research, 49: 47-58. 
[28] Otsuka H. 2017. Capacity developments in the world steel industry. Tech. rep.. OECD. Available at: oe.cd/steelcapacity.

[29] Pedroso CB, da Silva AL \& Tate WL. 2016. Sales and Operations Planning (S\&OP): Insights from a multi-case study of Brazilian Organizations. International Journal of Production Economics, 182: 213-229.

[30] Pochet Y \& Wolsey LA. 2006. Production planning by mixed integer programming. Springer.

[31] SEOng D \& SuH MS. 2012. An integrated modelling approach for raw material management in a steel mill. Production Planning \& Control, 23(12): 922-934.

[32] Shabani N \& Sowlati T. 2016. A hybrid multi-stage stochastic programming-robust optimization model for maximizing the supply chain of a forest-based biomass power plant considering uncertainties. Journal of Cleaner Production, 112: 3285-3293.

[33] Taşkin ZC, AĞrali S, Ünal AT, Belada V \& Gökten-Yilmaz F. 2015. Mathematical Programming-Based Sales and Operations Planning at Vestel Electronics. Interfaces, 45(4): 325-340.

[34] Thomé AMT, Scavarda LF, Fernandez NS \& Scavarda AJ. 2012. Sales and operations planning: A research synthesis. International Journal of Production Economics, 138(1): $1-13$.

[35] Thomé FS, Binato S, Pereira MV, Campodónico N, Fampa MH \& COSTA JR LCD. 2013. Decomposition approach for generation and transmission expansion planning with implicit multipliers evaluation. Pesquisa Operacional, 33: 343-359. Available at: http://www.scielo.br/scielo.php?script=sci_arttext\& pid $=$ S0101-74382013000300002\&nrm=iso.

[36] TuOmikangas N \& Kaipia R. 2014. A coordination framework for sales and operations planning (S\&OP): Synthesis from the literature. International Journal of Production Economics, 154: 243-262.

[37] VAn SLYKe RM \& WeTS R. 1969. L-shaped linear programs with applications to optimal control and stochastic programming. SIAM Journal on Applied Mathematics, 17(4): 638-663.

[38] WANG JZ, Hsieh ST \& HSU PY. 2012. Advanced sales and operations planning framework in a company supply chain. International Journal of Computer Integrated Manufacturing, 25(3): 248-262.

[39] Wang S, Gangammanavar H, EkşioĞLu S \& Mason SJ. 2020. Statistical estimation of operating reserve requirements using rolling horizon stochastic optimization. Annals of Operations Research, 292(1): 371-397. 
[40] You F \& GRossmann IE. 2013. Multicut Benders decomposition algorithm for process supply chain planning under uncertainty. Annals of Operations Research, 210(1): 191211.

[41] Zhulanova J \& Zhulanov K. 2014. Coordination between production and sales planning in an oil company based on Lagrangean Decomposition. Master's thesis. Norwegian School of Economics.

\section{How to cite}

ALMEIDA JFF \& CONCEIÇÃo SV. 2021. A decomposition approach for the two-stage stochastic supply network planning in light of the rolling horizon practice. Pesquisa Operacional, 41 (s1): e234451. doi: 10.1590/0101-7438.2021.041s1.00234451. 